\title{
Digital ulcers and multiple amputations in a systemic sclerosis patient
}

\author{
Laura Groseanu ${ }^{1,2}$, Ina Cambur', Andra Balanescu ${ }^{1,2}$, Ruxandra Ionescu ${ }^{1,2}$ \\ ${ }^{1}$ Department of Internal Medicine and Rheumatology, "Sf. Maria" Clinical Hospital, Bucharest, Romania \\ 2"Carol Davila" University of Medicine and Pharmacy, Bucharest, Romania
}

\begin{abstract}
We are presenting the case of a 48 years old female diagnosed at the age of 28 with diffuse cutaneous systemic sclerosis. During the course of disease, despite the vasodilator treatments and immunosuppression for visceral involvement, the patient developed multiple infected digital ulcers both in the upper limbs and in the lower limbs, which complicated with wet gangrene, requiring transmetatarsal amputations. At that time, a macrovascular disease or a prothrombotic condition were excluded. Patient also developed infected nondigital lower extremity ulcers, which slowly healed after Alprostadil treatment. The evolution of our patient's disease demonstrate that the management of scleroderma vasculopathy represent a challenge and a multidisciplinary approach is needed.
\end{abstract}

Keywords: systemic sclerosis, digital ulcers, nondigital ulcers, critical digital ischemia, amputation

\section{INTRODUCTION}

Digital ulcers (DUs) are a major clinical problem in systemic sclerosis (SSc), occurring in $~ 30 \%$ of the patients each year (1). DUs not only impact significantily on patients quality of life and hand function, but are also biomarker of internal organ involvement and of disease severity (2). Chronic ulcers can become infected, resulting in gangrene, osteomyelitis and amputation (1). Nondigital lower limb ulcers are less frequent in systemic sclerosis, with a prevalence estimated to $4 \%$. In this case, any concomitent pathology (specifically, large vessel disease, vasculitis, coagulopathy, thromboembolism and smoking) that could be contributory should be identified (3). Management of DUs in systemic sclerosis includes nonpharmacologic, pharmacologic, and surgical intervention. There are a number of drug therapies avalaible to prevent (e.g. phosphodiesterase type- 5 inhibitors and endothelin receptor- 1 antagonists) and treat (e.g. intravenous iloprost) DUs (2). The effect of these drugs on nondigital ulcers in systemic sclerosis has not been studied.

\section{CASE PRESENTATION}

We are presenting the case of a 48 years old female, nonsmoker, who has as a pathological personal history: essential hypertension stage II in treatment with enzyme conversion inhibitor and thiazide diuretic and hypercholesterolemia in statin therapy.

The onset of rheumatic disease was in 2008 with arthralgia in small joints of the hands, Raynaud's phenomenon, DUs in the third fingertips of the hands and dysphagia. Physical examination revealed scleroderma face, pitting scars, puffy fingers, skin thickening proximal to the elbows with a modified Rodnan skin score of 31 . She presented a positive anti Scl-70 (antitopoisomerase) antibody and the nailfold capillaroscopy showed multiple avascular areas and dismorphic capillaries. Barium swallow X-rays was performed which described moderate gastroesophageal reflux. Baseline cardiopulmonary tests were normal. Therefore, the diagnosis was diffuse cutaneous systemic sclerosis and the treatment given was with Methotrexate up to $20 \mathrm{mg} /$ week, calcium channel blocker, aspirin, proton-pump inhibitor and non-steroidal inflammatory. 
Two years after the diagnosis, despite the immunosuppressive and vasodilatator treatment, the patient developed critical digital ischemia in the I-IV left foot fingers with a DU in the left toe which was infected with Staphylococcus Aureus. At that moment a macrovascular disease or prothrombotic condition were excluded (Doppler ultrasoud scan, conventional angiography, ECG, echocardiogram, immunology testing for vasculitis and antiphospholipid syndrome were all normal). She was treated with anticoagulants, intravenous antibiotic, intravenous Pentoxiphylline and opioid based analgesia. There was no clinical improvement, the patient developed wet gangrene which required left transmetatarsal amputation. The Methotrexate treatment was discontinued resumed one month after surgery.

In 2011, one year after surgical intervention, the patient developed multiple DUs in the hands. At that moment, the treatment with Methotrexate was discontinued due to ineffectiveness and Alprostadil was initiated. The therapeutic response was initially good, then in 2014 the patient developed new DUs in the hands, which at that time did not respond to Alprostadil. Under these conditions, Alprostadil treatment was stopped and Bosentan therapy was started up to $250 \mathrm{mg}$ /day.

In 2015 the patient presented with progressive dyspnoea on exertion, that had started two months prior to admission. Chest radiography revealed signs of increased interstitial markings in both lungs. Pulmonary function tests showed decreased lung volumes $(\mathrm{FVC}=72 \%, \mathrm{FEV} 1=76 \%)$ and a decreased carbon monoxide diffusion capacity (66\%). Computerized tomography scan confirmed the presence of a bilateral interstitial lung disease with honeycomb appearance in both lung fields. Because of the interstitial lung disease, immunosuppressive therapy with $\mathrm{Cy}$ clophosphamide $600 \mathrm{mg}$ intravenous monthly was started. Two months later, in the presence of vasodilator treatment with Bosentan, the patient developed critical digital ischemia in the I-III right foot fingers with a DU in the right toe which was infected with Staphylococcus aureus. Under these conditions, Cyclophosphamide therapy was discontinued due to infection and Bosentan treatment was also discontinued due to inefficiency. Alprostadil was restarted, combined with anticoagulants, intravenous antibiotic and opioid based analgesia. Despite this treatment, the patient developed wet gangrene and for this reason transmetatarsal amputation was performed.

Subsequently, between July and August 2016, the patient developed multiple DUs in the hands, an ulcer in the right heel which was infected with Pseudomonas aeruginosa and an ulcer in the left crippled of amputation which was infected with Staphyloccocus Aureus. X-ray were performed showing the absence of osteolysis zones, the appearance being not suggestive for osteitis. The treatment included wound care (autolytic debridement with moisture-retentive dressing such as hydrogel and hydrocolloids, topical antimicrobials and antiseptics), Alprostadil, calcium channel blocker, anticoagulant, anti-platelet, antibiotic and analgesia therapy. After six months the DUs from the hands had completely healed with no new DUs or necrosis of her fingers. Regarding the ulcers of right heel and left crippled of the ampu-
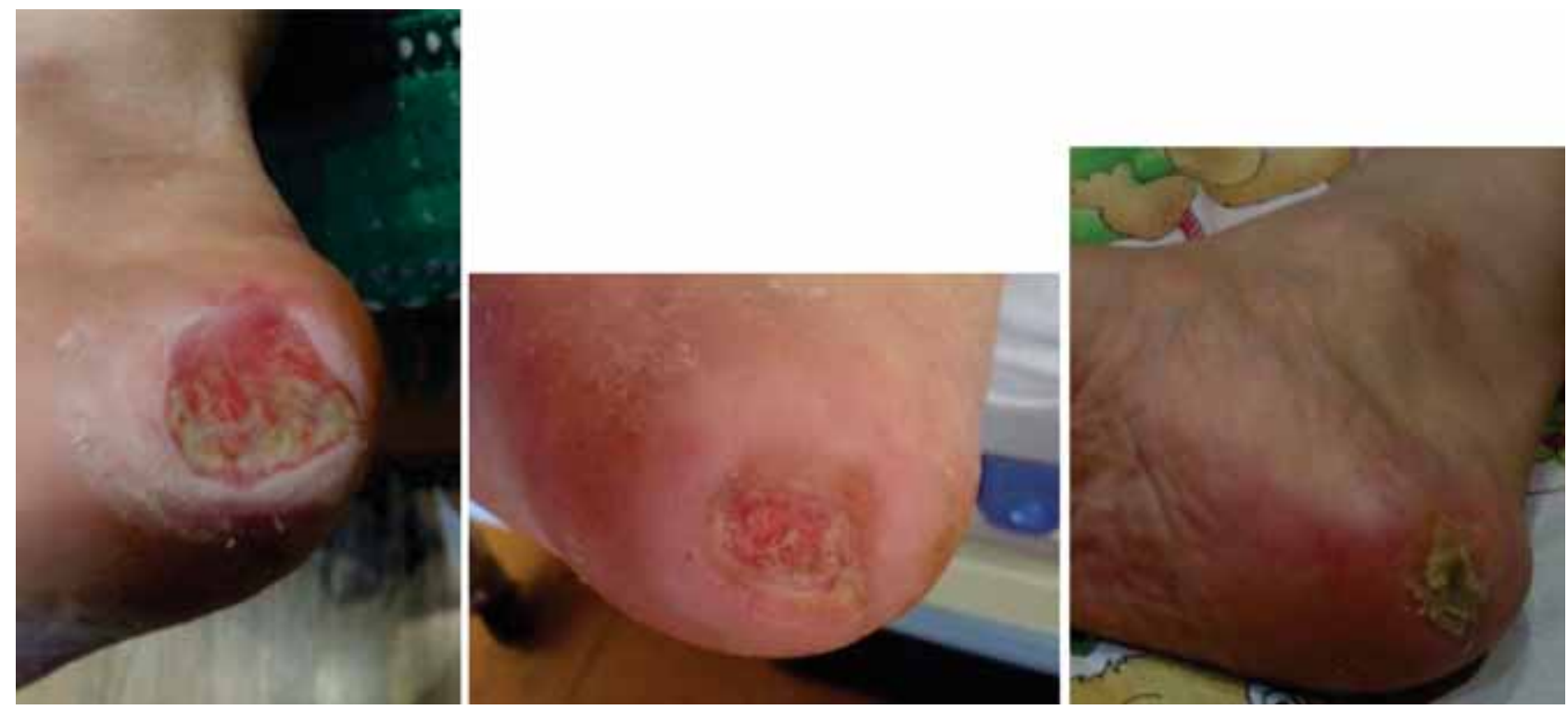

FIGURE 1. Evolution of the ulcer in the right heel - july 2016, july 2017 and september 2017 


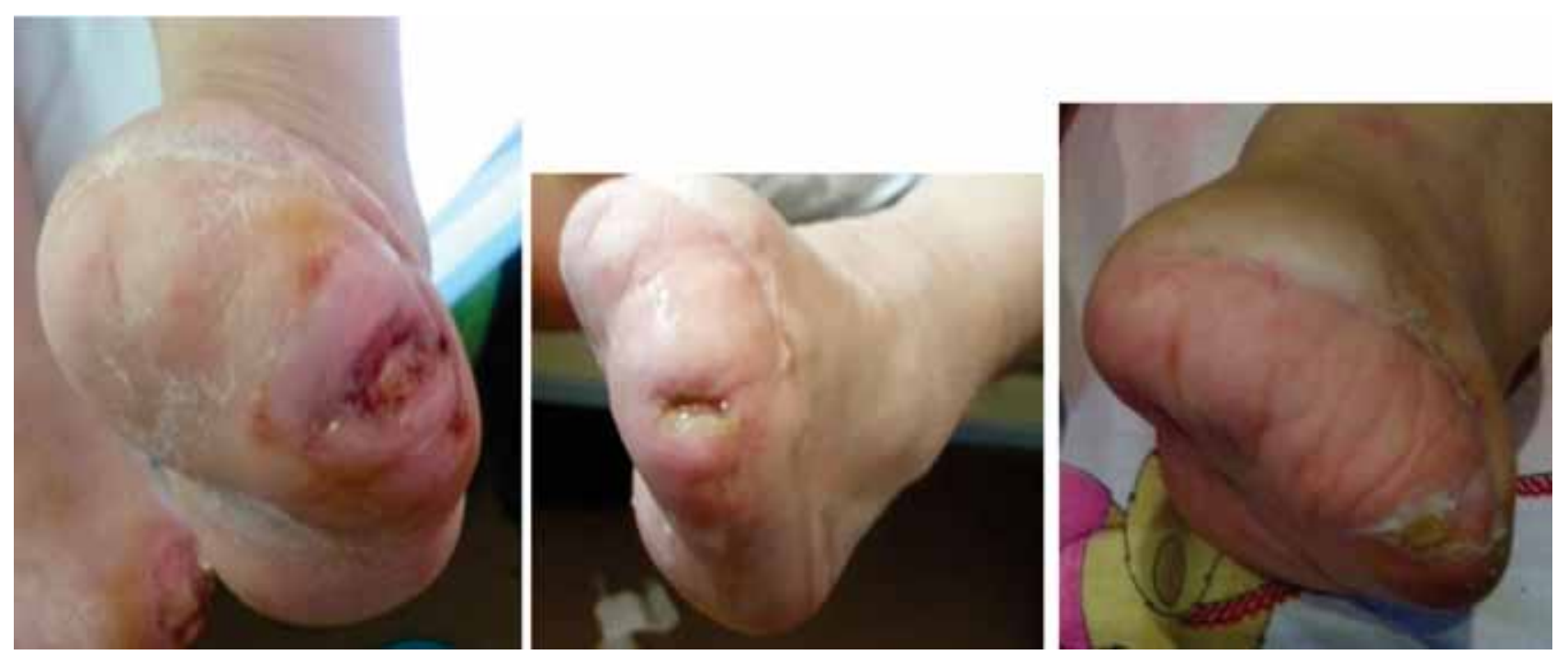

FIGURE 2. Evolution of the ulcer in the left crippled of the amputation - july 2016, july 2017 and september 2017

tation the evolution was tremendous with very slow healing (Fig. 1 and Fig. 2).

Particularities of this case consists in the development of multiple recurrent ulcers (both in the upper and lower limbs) despite the vasodilatator treatment with Bosentan, but also despite the immunosuppressive therapy for visceral involvement. Another particulary was the development of lower limb ulcers in the absence of macrovascular disease or a prothrombotic condition. Besides these, recurrent infections of the ulcers caused temporary discontinuation of immunosuppressants.

\section{DISCUSSIONS}

DUs have a significant burden for patients with systemic sclerosis: more than $60 \%$ of them develop ulcers, $30 \%$ of them are severe, multiple, recurrent, very paintful, slowly healing of and have a considerable functional impact (4). The considerable burden is due not only to the DUs itself but also to its major complications: infection of soft tissue (up to $40 \%$ ) or osteomyelitis (up to 4\%), gangrene (up to $30 \%$ ) that can lead to amputation (4). DUs are not only a common clinical feature of SSc, but can also be regarded as a severity biomarker of the disease. A recent analyse of EUSTAR cohort, concluded that DUs are predictive for new DUs and associated with higher risk of developing pulmonary hypertension, cardiovascular events and higher mortality (5).

SSc is mainly a disease of the endothelial cells, an imbalance between vasodilators and vasoconstrictors mediators leads to repeated episodes of ischemia and reperfusion with tissular hypoxia, releasing free oxygen radicals; initially the vasculopathy is functional but then it becomes organic with capillary loss, intimal fibrosis and local coagulation anomalies $(6,7)$. But why our patient develop some many episodes of multiple, recurrent, slowly healing of digital ulcers in upper and lower limbs? In this case, other causes should be looked for as: atherosclerosis, macrovascular disease related to systemic sclerosis, mainly involving ulnar arthery, protrombotic conditions, coagulation anomalities (antiphospholipid syndrome, thrombophilia etc) or overlap with other autoimmune diseases (crioglobulinemia, vasculitis, systemic lupus erythematosous) $(3,6,8,9)$. Our patient did not have any cardiovascular risk factors, except for hypertension and other conditions mentioned were ruled out in the beginning. Little is known about lower limb ulcers in systemic sclerosis; their prevalence is about $4 \%$, compared to other systemic sclerosis patients they have a significant higher association of protrombotic conditions as antiphospholipid syndrome (50\% vs $3.3 \%-12 \%$ ), homo/heterozygote mutations of homocysteine genes (15\% vs $10 \%, 60 \%$ vs $49 \%$ )or rPAI- $1(40 \%$ vs $5-6.8 \%)(3,9)$.

Can we predict DUs? There are some patients at risk: early onset of Raynaud, males, antiSCL70 positive patients, the smokers, patients with pulmonary hypertension or inflammatory syndrome, previous DUs (10). Nailfold capillaroscopy also has an important predictive role: worsening of capillaroscopic pattern and specific capillaroscopy scores have been associated with higher risk of DUs. In the case presented the disease started in her 30s, she had antiSCL70 autoantibodies, inflammatory syndrome and a late capillaroscopic pattern just from disease onset. 
The managment of digital ulcers should be multidisciplinary, combining patient education, preventive and curative treatments and local therapy; surgical treatment should be the last option.

Non- pharmacologic intervention - patients should be advised to avoid cold exposure, emotional stress, drugs that could induce vasospasm (betablockers, nasal decongestants with ephedrine, antimigraine drugs like Sumatriptane, chemotherapy as cysplatine), quit smoking and limit coffee consumption, wash hands with antibacterial soap, keep good skin moisturizing, avoid hand trauma (including manicure or repetitive hand working as typing), to have an antioxidants rich diet, use protective digital cap, orthopedic shoes $(12,13)$.

The treatment of a digital ulcer starts with the evaluation of its severity. Localization, size, pain, type of exudate, borders, bed of the lesion, perilesional tissue, exposure of underneath tissue or bone, autoamputation need to be evaluated. Specific investigations are also done in this step to assess the DUs complications: radiographs (to rule out osteomyelitis), cultures (to rule out soft tissue infection) (14). This step is important in order to choose a therapeutic strategy: the type of local treatment, the need for painkillers or antibiotics, the right class or combination of vasodilators.

The second step of the strategy is local treatment. General rules include: sterile washing with isotonic saline solution, hypoclorite and saline solution again, necrotic tissue debridment. Hydrogels and semi-occlusive wound dressings prevent evaporative water loss and retain warmth, which improves wound healing (in patients with infected ulcers, like our case the most commonly used dressings are those with silver nitrate) (15) .

In the third step, specific investigations are done to rule out associated condition, previously mentioned. Especially in patients with severe, multiple, recurrent DUs or with ulcers in the lower limbs, they should be reassessed periodically.

\section{Pharmacological therapy}

Despite the substantial impact that DUs have on function and life quality of SSc patients, currently there is no widely accepted therapeutic algorithm.

Dyhidropiridine type calcium channel blockers are the first choice for Raynaud treatment, but their effect on DUs prevention and healing is not significant (12).
Prostanoids are potent vasodilators and also inhibit platelet aggregation and vascular smooth muscle cell proliferation. Data reporting efficacy of these drugs for Raynaud treatment comes mostly from pulmonary hypertension studies, but only some of the studies reports benefits for DUs healing and prevention. Intravenous prostanoid therapy has been reported to significantly improve DUs healing and reduce the number of new DUs $(16,17)$. Continous iv Epoprostenol and sc Treprostenil administration have also been associated with a reduction of new DUs $(18,19)$. Unfortunately they are not reimbursed for DUs treatment.

Endothelin receptor antagonists are potent vasodilators and inhibit smooth muscle cells and fibroblasts proliferation. Two studies, RAPIDS-1 and RAPIDS-2, found Bosentan to be useful in the prevention of the onset of new lesions in SSc patients who had already experienced multiple DUs but seems to have no effect in healing of the existing ulcers (12). In the case presented, 6 month of Bosentan had no effect on DUs prevention and the patient developed some side effects (headache, arthralgias).

Phosphodiesterase type-5 inhibitors inhibit the degradation (and therefore increase the bioavailability) of cyclic GMP, with subsequent vasodilatation. In a meta-analysis of DU, PDE5 inhibitors were associated with DU healing and improvement (12). Like prostanoids, they could not be used in our case because of the lack of reimbursement.

Few randomized, placebo controlled trials, on a limited number of patients reported adjuvant vasodilator effects of Vitamin E gel and topical nitrates $(20,21)$

\section{Other drugs}

In patients with multiple, severe, recurrent DUs, like our case, backup therapies might be used. N-acetylcysteine(22), alpha-blockers (prazosin) (23), selective uptake serotonine inhibitors (24) might be useful. Their efficacy in healing and prophylaxy of DUs is modest and side effects limit their use. None of them was used in our case. Although there is a strong therapeutic rationale for the role of angiotensin-converting enzyme inhibition in DU disease, including use as vascular remodelling agents, there is no current evidence base to support this intervention. They might be useful for symptomatic improvement of Raynaud (25), but long term treatment with quinapril was not associated with a significant reduction in the number of new Dus (26). Non-phar- 
maceutical treatment modalities as hyperbaric oxygen chamber, negative pressure therapy, intermittent compression, acoustic pressure might also be used but they are not reimbursed and difficult to access (27). There is insufficient evidence at present to recommend immunosuppressive therapy for Dus treatment $(28,29)$ and infected DUs are a relative contraindication. In the case presented, altghough immunosuppression was started early it was not effective in DUs prevention and it was often stopped temporarily due to infection of DUs or prior to surgery. Although many clinicians prescribe antiplatelet therapy for DUs (and severe Raynuad), there is a lack of good evidence base to support this intervention (30). Low-molecular-weight heparin was not associated with a significant difference in new DUs (31). The fact that many patients with SSc have gastrointestinal involvement with a propensity to bleeding should be taken into account when assessing the risk-to-benefit ratio for any individual patient. Anticoagulants were beneficial in our case especially for lower limb ulcer, healing was promoted when therapy was restarted. In a randomized trial, atorvastatin treatment was associated with a reduction in the number of new DUs(32). Further research is required before statin therapy can be recommended widely. Sympathectomy, injections with botulinum

\section{REFERENCES}

1. Denton C.P., J.H. Korn. Digital ulceration and critical digital ischaemia in scleroderma, Scleroderma Care Res, 2003, vol. 1 (pg. 12-6)

2. Hughes M., AlL Herrick A.I. Digital ulcers in systemic sclerosis. Rheumatology 2017; 56:14-25

3. Shanmugam J.V.K. et al. "Lower extremity ulcers in systemic sclerosis: features andresponse to therapy," International Journal of Rheumatology, vol. 2010, Article ID747946, 8 pages, 2010.

4. Christopher P. Denton Demographic, clinical and antibody characteristics of patients with digital ulcers in systemic sclerosis: data from the DUO Registry Ann Rheum Dis 2012; 71(5): 718-721

5. Mihai C. et al. Digital ulcers predict a worse disease course in patients with systemic sclerosis. Ann Rheum Dis. 2016; 75(4):681-6.

6. Digital ulcers in systemic sclerosis Rheumatology (Oxford). 2016; 56(1):14-25

7. Suliman Y.A. Defining skin ulcers in systemic sclerosis sytematic literature review and proposed World Scleroderma Foundation Definition, J Scleroderma Relat Disord 2017, 2(2) 115-120

8. Belch J.J. et al. Macrovascular disease in systemic sclerosis: the tip of an iceberg? Rheumatology (Oxford).2008; 47Suppl 5:v16-7

9. Steen V. Digital ulcers - Overt vascular disease in systemic sclerosis Rheumatology, 2009(48):3, iii19-iii24

10. Sunderkötter $\mathbf{C}$. et al. Comparison of patients with and without digital ulcers in systemic sclerosis: detection of possible risk factors Br J Dermatol. 2009; 160(4):835-43

11. Sebastiani M. et al. Capillaroscopic skin ulcer risk index: a new prognostic tool for digital skin ulcer development in systemic sclerosis patientsArthritis Rheum. 2009; 61(5):688-94 toxin, autologous fat grafting and stem cell transplant have been also reported to promote DUs healing for selected cases in small case series (12).

\section{The role of the surgeon for DUs}

A surgical consult may be necessary in the case of failure of the medical approaches; a surgical opinion should be sought whenever a macrovascular involvement is suspected. Therapeutic strategy should be as conservative as possible with necrotic disuse debridment, excision of soft tissue calcification, sympathectomy. The time of healing is thought to be longer (33). Unfortunatelly, in our case surgery was radical, with both forefoot amputation due to gangrene evolution and severe pain that could not be controlled with first and second-line painkillers.

In conclusion, DUs are a common and disabling manifestation of the underlying vascular disease in SSc. Patient education is crucial, and patients should be managed within a dedicated multidisciplinary team. Although there are a number of effective treatments for DUs, side effects are not uncommon, and patients may develop new DUs despite treatment. Future research is warranted to optimize the management of DUs, including (but not limited to) combination therapies and the development of locally acting treatments.

12. Tingey T. et al. Meta-analysis of healing and prevention of digital ulcers in systemic sclerosis Arthritis Care Res (Hoboken). 2013; 65(9):1460-71

13. Gualtierotti R. et al. Digital ulcer management in patients with systemic sclerosis. OA Arthritis 2014-18; 2(1):2.

14. Matucci-Cerinic M. et al. Digital ulcers and outcomes assessment in scleroderma Rheumatology 2008(47):46-v47

15. Fonder M.A. et al. Treating the chronic wound: A practical approach to the care of nonhealing woundsand wound care dressings. J Am AcadDermatol. 2008; 58:185-206.

16. Wigley F.M. et al. Intravenous iloprost treatment of Raynaud's phenomenon and ischemic ulcers secondary to systemic sclerosis J Rheumatol 1992; 19:1407-14

17. Wigley F.M. et al. Intravenous iloprost infusion in patients with Raynaud phenomenon secondary to systemic sclerosis. A multicenter, placebo-controlled, double-blind study. Ann Intern Med 1994; 120:199-206

18. Badesch D.B. et al. Continuous intravenous epoprostenol for pulmonary hypertension due to the scleroderma spectrum of disease. A randomized, controlled trial. Ann Intern Med 2000; 132:425-34

19. Chung L. et al. A pilot trial of treprostinil for the treatment and prevention of digital ulcers in patients with systemic sclerosis. J Am Acad Dermatol 2006; 54:880-2

20. Hummers L.K. et al. A multi-centre, blinded, randomized, placebo-controlled, laboratory-based study of MQX-503,a novel topical gel formulation of nitroglycerine, in patients with Raynaud phenomenon. Ann Rheum Dis. 2013; 72:1962-1967 
21. Fiori G. et al. Vitamin $E$ gel reduces time of healing of digital ulcers in systemic sclerosis. Clin Exp Rheumatol. 2009;

27(3 Suppl 54):51-4.

22. Correa M.J. et al. Oral $\mathrm{N}$-acetylcysteine in the treatment of Raynaud's phenomenon secondary to systemic sclerosis: A randomized, double-blind, placebo-controlled clinical trial Revista Brasileira de Reumatologia 2014; 54(6):452-458

23. Wollerstheim D. Double-blind, placebo-controlled study of prazosin in Raynaud's phenomenon Clin Pharmacol Ther. 1986; 40(2):219-25

24. Lukac J. et al. Effect of ketanserin on Raynaud's phenomenon in progressive systemic sclerosis: a double-blind trial DrugsExp Clin Res. 1985; 11(9):659-63

25. Dziaodzio M. et al. Losartan therapy for Raynaud's phenomenon and scleroderma: clinical and biochemical findings in a fifteenweek, randomized, parallel-group, controlled trial. Arthritis Rheum. 1999; 42:2646-2655

26. Gliddon A.E. et al. Prevention of vascular damage in scleroderma and autoimmune Raynaud's phenomenon: a multicenter, randomized, double-blind, placebo-controlled trial of the angiotensin-converting enzyme inhibitor quinapril. Arthritis Rheum. 2007; 56(11):3837-46.
27. Moran M.E. Scleroderma and evidence based non-pharmaceutical treatment modalities for digital ulcers: a systematic review. J Wound Care. 2014; 23(10):510-6.

28. Khor C. et al. Rituximab for refractory digital infarcts and ulcers in systemic sclerosis Clin Rheumatol 2014; 33:1019-20

29. Fernandes das Neves et al. Treatment of systemic sclerosis with tocilizumab. Rheumatology 2015; 54:371-2

30. Beckett V.L. et al. Trial of platelet-inhibiting drug in scleroderma: double-blind study with dipyridamole and aspirin. Arthritis Rheum 1984; 27:1137-43.

31. Denton C.P. et al. Long-term low molecular weight heparin therapy for severe Raynaud's phenomenon: a pilot study. Clin Exp Rheumatol 2000;18: 499-502.

32. Abou-Raya A. Statins: potentially useful in therapy of systemic sclerosis-related Raynaud's phenomenon and digital ulcers. J Rheumatol 2008; 3:1801-8

33. Bogogh E.R. et al. Surgery of the hand in patients with systemic sclerosis: outcomes and considerations. J Rheumatol. 2005; 32(4):642-8. 